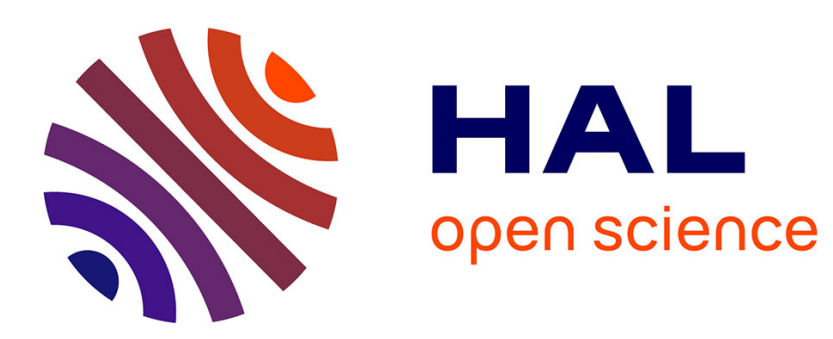

\title{
Collision operators for Stark line shapes: When does the unified theory fail?
}

Joël Rosato, Hubert Capes, Roland Stamm

\section{To cite this version:}

Joël Rosato, Hubert Capes, Roland Stamm. Collision operators for Stark line shapes: When does the unified theory fail?. High Energy Density Physics, 2013, http://dx.doi.org/10.1016/j.hedp.2013.04.005. hal-00830431

\section{HAL Id: hal-00830431 \\ https://hal.science/hal-00830431}

Submitted on 5 Jun 2013

HAL is a multi-disciplinary open access archive for the deposit and dissemination of scientific research documents, whether they are published or not. The documents may come from teaching and research institutions in France or abroad, or from public or private research centers.
L'archive ouverte pluridisciplinaire HAL, est destinée au dépôt et à la diffusion de documents scientifiques de niveau recherche, publiés ou non, émanant des établissements d'enseignement et de recherche français ou étrangers, des laboratoires publics ou privés. 


\title{
Collision operators for Stark line shapes: when does the unified theory fail?
}

\author{
J. Rosato, H. Capes, R. Stamm \\ Aix-Marseille Université, CNRS, PIIM UMR 7345, F-13397 Marseille Cedex 20, France
}

\begin{abstract}
It is shown that the unified theory for Stark broadening leads to incorrect results when correlated emitter-perturber collisions are present. With a kinetic theory treatment, we propose an extension of the model able to account for such correlations. The treatment presents analogies with renormalization techniques and the resulting collision operator has a structure similar to that obtained within the unified theory. We illustrate the applicability of the model through calculations of hydrogen line shapes in ideal cases.
\end{abstract}

Keywords: Line shapes, Stark broadening

PACS: $32.60 .+\mathrm{i}, 32.70 . \mathrm{Jz}$

\section{Introduction}

In plasma spectroscopy, it has been a long-standing challenge to accurately describe Stark-broadened line shapes. Stark broadening arises when dealing with spectral lines in high-density medium, for diagnostics or for opacity calculations [1], but also in moderately dense plasmas such as in magnetic fusion experiments [2]. The problem consists in a correct description of the atomic dipole autocorrelation function in the presence of the plasma microscopic electric field. The so-called "standard model" consists in assuming the ions motionless during the dipole correlation time and, at the same time, assuming the electrons moving rapidly. Their contribution is described through a non-Hermitian part in the Hamiltonian, the collision operator. Ion dynamics effects have been extensively investigated, using kinetic theory methods [3], numerical simulations $[4,5,6,7]$ or ad hoc models such as the frequency fluctuation model [8]. On the other hand, there have been several attempts in the past to retain static electron effect, i.e., incomplete collisions, in the collision operator. A first-principles-based treatment is provided by the "unified theory" $[9,10]$. This formalism relies on a set of equations similar to those used in kinetic theory within the BogoliubovBorn-Green-Kirkwood-Yvon (BBGKY) hierarchy and uses an approximation

Email address: joel.rosato@univ-amu.fr (J. Rosato) 
of weak-coupling type for solving them, based on the assumption that strong collisions are rare events. This assumption amounts to considering the dimensionless parameter $g=N b_{W}^{3}$ (with $N, b_{W}$ being the density of perturbers and the Weisskopf radius, respectively) small compared to unity. Recent modeling efforts have been undertaken by our group to analyze the limits of applicability of this model [11]. Here, we report on the current status of this work. It is shown that the unified theory can lead to incorrect results (in particular with unphysical dips at the line center) even for rare strong collisions. This discrepancy occurs when correlations between the collisions are present. By applying a resummation procedure to the hierarchy equations, we propose an extension of the unified theory able to account for such correlations. Section 2 gives an overview of the unified theory and Sec. 3 addresses the description of correlated collisions. Calculations of hydrogen spectral profiles, with comparisons to numerical simulations, are presented in Sec. 4.

\section{Unified theory}

We give here a brief overview of the BBGKY approach applied to the line broadening problem, following the early works reported on in Refs. [9, 12, 13]. An atom immersed in a set of $\mathcal{N}$ charged particles is considered. For simplicity, we assume one-component plasma and we consider the particles evolving along straight lines and generating a Debye electric field. A generalization accounting for correlations between the perturbers in a rigorous way can be developed following [12]. A Stark line shape $I(\omega)$ is written as

$$
I(\omega)=\frac{1}{\pi} \operatorname{Re} \sum_{\boldsymbol{\varepsilon}}\left\langle\left\langle\mathbf{d} \cdot \varepsilon\left|\tilde{\Phi}_{0}(-i \omega)\right| \rho \mathbf{d} \cdot \varepsilon\right\rangle\right\rangle .
$$

Here, the double ket notation for Liouville space $|\ldots\rangle\rangle$ has been used, $\rho$ is the atomic density operator, $\mathbf{d} \cdot \boldsymbol{\varepsilon}$ is the dipole projected onto the polarization vector $\varepsilon$, and $\tilde{\Phi}_{0}(-i \omega)=\int_{0}^{+\infty} d t e^{i \omega t} \Phi_{0}(t)$ is the Laplace transform of the evolution operator averaged over the perturber trajectories assuming classical paths. The latter is obtained from the following definitions with $p=1 \ldots \mathcal{N}$

$$
\begin{aligned}
\Phi_{p}(1 \ldots p ; t) & =\int d(p+1) \ldots d \mathcal{N} f_{\mathcal{N}}(1 \ldots \mathcal{N}) U(1 \ldots \mathcal{N} ; t), \\
\Phi_{0}(t) & =\int d 1 \ldots d \mathcal{N} f_{\mathcal{N}}(1 \ldots \mathcal{N}) U(1 \ldots \mathcal{N} ; t) .
\end{aligned}
$$

Here, $1 \ldots \mathcal{N}$ stand for the phase space coordinates $\left(\mathbf{r}_{1}, \mathbf{v}_{1} \ldots \mathbf{r}_{\mathcal{N}}, \mathbf{v}_{\mathcal{N}}\right)$ of the perturbers, $d 1 \ldots d \mathcal{N}$ are the corresponding volume elements, $f_{\mathcal{N}}$ is the $\mathcal{N}$-particle phase space distribution, and $U$ is the atomic evolution operator. It obeys the Liouville equation

$$
\left\{\frac{\partial}{\partial t}+i L_{0}+\sum_{j=1}^{\mathcal{N}}\left[\mathbf{v}_{j} \cdot \frac{\partial}{\partial \mathbf{r}_{j}}+i V(j)\right]\right\} U=0
$$


with the initial condition $U(t=0)=1$. All the quantities present in Eq. (4) are operators acting in the atomic Liouville space formed by the dyadics $|a b\rangle\rangle \equiv|a\rangle|b\rangle$. The term $L_{0}$ is the Liouvillian accounting for the atomic energy level structure and $V(j)=V\left(\mathbf{r}_{j}\right)=-\mathbf{d} \cdot \mathbf{E}\left(\mathbf{r}_{j}\right)$ denotes the Stark term resulting from the electric field due to the $j$-th perturber.

Equation (2) provides generalizations of the reduced phase space distributions, which account for the presence of the atom. It is customary to introduce a cluster expansion ( $t$ is not written explicitly)

$$
\left\{\begin{aligned}
\Phi_{1}(1)= & f_{1}(1) \Phi_{0}+\Gamma_{1}(1) \\
\Phi_{2}(1,2)= & f_{2}(1,2) \Phi_{0}+f_{1}(1) \Gamma_{1}(2)+f_{1}(2) \Gamma_{1}(1)+\Gamma_{2}(1,2) \\
\Phi_{3}(1,2,3)= & f_{3}(1,2,3) \Phi_{0}+f_{2}(1,2) \Gamma_{1}(3)+f_{2}(1,3) \Gamma_{1}(2)+f_{2}(2,3) \Gamma_{1}(1) \\
& +f_{1}(1) \Gamma_{2}(2,3)+f_{1}(2) \Gamma_{2}(1,3)+f_{1}(3) \Gamma_{2}(1,2)+\Gamma_{3}(1,2,3) \\
\ldots &
\end{aligned}\right.
$$

The $\Gamma$ s denote generalized correlation functions. They obey a hierarchy of equations

$$
\begin{aligned}
& \left\{\frac{\partial}{\partial t}+i L_{0}+\sum_{j=1}^{p}\left[\mathbf{v}_{j} \cdot \frac{\partial}{\partial \mathbf{r}_{j}}+i V(j)\right]\right\} \Gamma_{p}(1 \ldots p) \\
& =-i \sum_{j=1}^{p} f_{1}(j) V(j) \Gamma_{p-1}(1 \ldots j-1, j+1 \ldots p)-i \mathcal{N} \int d(p+1) V(p+1) \Gamma_{p+1}(1 \ldots p+(16))
\end{aligned}
$$

with the initial condition $\Gamma_{p}(1 \ldots p ; t=0)=0$ for $p \geq 1$. It has been assumed that the phase space distributions are space independent and factorize as $f_{p}(1 \ldots p)=f_{1}(1) \ldots f_{1}(p)$. Also, the thermodynamic limit $(\mathcal{N} \rightarrow \infty, \mathcal{V} \rightarrow \infty$, $\mathcal{N} / \mathcal{V}=$ constant with $\mathcal{V}$ being the volume of the system) is assumed. In Eq. (6), by convention $\Gamma_{0} \equiv \Phi_{0}$ and $\Gamma_{-1} \equiv 0$.

Originally, the unified theory was developed for applications in cases where the collisions are uncorrelated. The treatment involves equations for $\Phi_{0}$ and $\Gamma_{1}$ only, setting $\Gamma_{2} \equiv 0$ :

$$
\left(\frac{\partial}{\partial t}+i L_{0}\right) \Phi_{0}=-i \mathcal{N} \int d 1 V(1) \Gamma_{1}(1)
$$

and

$$
\left[\frac{\partial}{\partial t}+i L_{0}+\mathbf{v}_{1} \cdot \frac{\partial}{\partial \mathbf{r}_{1}}+i V(1)\right] \Gamma_{1}(1)=-i f_{1}(1) V(1) \Phi_{0} .
$$

Equation (8) can be solved formally by using the propagator of the atom under the influence of one perturber, $Q(1, t)$. It obeys the following equation

$$
\left[\frac{\partial}{\partial t}+i L_{0}+i V\left(\mathbf{r}_{1}+\mathbf{v}_{1} t\right)\right] Q(1, t)=0,
$$

with the initial condition $Q(1, t=0)=1$, and it is proportional to a timeordered exponential (Dyson series)

$$
Q(1, t)=e^{-i L_{0} t} \mathcal{T} \exp \left[-i \int_{0}^{t} d \tau e^{i L_{0} \tau} V\left(\mathbf{r}_{1}+\mathbf{v}_{1} \tau\right) e^{-i L_{0} \tau}\right]
$$


$\mathcal{T}$ being the time-ordering operator. The correlation $\Gamma_{1}$ is obtained from a convolution

$$
\Gamma_{1}(1 ; t)=-i f_{1}(1) \int_{0}^{t} d \tau Q\left(\mathbf{r}_{1}-\mathbf{v}_{1} \tau, \mathbf{v}_{1}, \tau\right) V\left(\mathbf{r}_{1}-\mathbf{v}_{1} \tau\right) \Phi_{0}(t-\tau) .
$$

Inserting the solution Eq. (11) into the right-hand side of Eq. (7) provides a closed, integro-differential equation for the averaged evolution operator $\Phi_{0}(t)$. Its solution takes a simple form in the Laplace space:

$$
\tilde{\Phi}_{0}(s)=\left[s+i L_{0}+K(s)\right]^{-1} .
$$

Inserting this relation in Eq. (1) provides an analytical expression for the line shape. Decomposing the double bra and kets onto an appropriate base of the Liouville space indicates that the line shape can be written as a sum of generalized Lorentzian functions, whose widths are frequency-dependent and given by matrix elements of $K(s=-i \omega)$. The latter quantity is a collision operator that accounts for incomplete collisions. It is completely determined in terms of the propagator $Q$ and the interaction term $V$ :

$$
K(s)=\mathcal{N} \int_{0}^{\infty} d t e^{-s t} \int d 1 V\left(\mathbf{r}_{1}+\mathbf{v}_{1} t\right) Q(1, t) V(1) f_{1}(1) .
$$

\section{Correlated collisions}

The assumption $\Gamma_{2} \equiv 0$ considered in the unified theory is not valid in regimes where correlated collisions are present. Such correlations occur if, during the characteristic time for a binary collision which can be estimated as $\lambda_{D} / v \sim$ $\omega_{p}^{-1}$, where $v$ is the perturbers' thermal velocity, another perturber affects the atomic wavefunction. Such a perturbation occurs on a time scale of the order of the inverse matrix elements of the collision operator. A complete treatment of correlated collisions involving the infinite hierarchy Eq. (6) seems out of reach. In [11], we have proposed a simplification that uses the singular role of the $p+1$-th particle in the $p+1$-correlation present in the integral term of Eq. (6). The approach is inspired from diagrammatic techniques used in kinetic theory, in particular the "ring approximation", e.g., see Ref. [14]. For all $p \geq 1$, it is assumed that $p$-correlations are created or destroyed due to the $p$-th particle only. This simplification amounts to the replacement of the sums involving $V$ in Eq. (6) by their last term. The development is complex and will not be presented here; however, for details see Ref. [11]. The result is a nonlinear equation for the collision operator. It involves a formula similar to the result of the unified theory [Eq. (13)], with a modified propagator

$$
K(s)=\mathcal{N} \int_{0}^{\infty} d t e^{-s t} \int d 1 V\left(\mathbf{r}_{1}+\mathbf{v}_{1} t\right) Q_{\mathrm{eff}}(1, t) V(1) f_{1}(1) .
$$

The propagator obeys an integro-differential evolution equation

$$
\left[\frac{\partial}{\partial t}+i L_{0}+i V\left(\mathbf{r}_{1}+\mathbf{v}_{1} t\right)\right] Q_{\mathrm{eff}}(1, t)+\int_{0}^{t} d \tau M(\tau) Q_{\mathrm{eff}}(1, t-\tau)=0,
$$


with the initial condition $Q_{\text {eff }}(1, t=0)=1$. It generalizes the time-dependent Schrödinger Eq. (9), in the sense that correlations are retained. The memory kernel $M(t)$ is identical to the inverse Laplace transform of the collision operator. Equation (15) is interpretable as describing the evolution of the atom under the influence of one collision represented by the interaction term $V$, given a set of collisions occurring in its past history. These collisions act accumulatively and are taken into account by the kernel $M(t)$. The absence of this term in the unified theory [Eq. (9)] stems from the assumption $\Gamma_{2} \equiv 0$, which is valid provided the characteristic evolution time for $Q_{\text {eff }}$ is much shorter than the time between two collisions. In the case of a weakly correlated plasma, the evolution time is of the order of $\lambda_{D} / v$, so that a validity criterion is provided by the relation $\lambda_{D} \gamma / v \ll 1$ where $\gamma$ is a typical matrix element of the collision operator, e.g. estimated as $\gamma=N b_{W}^{2} v \ln \left(\lambda_{D} / b_{W}\right)$. This result indicates that the present extension of the unified theory allows one to explore regimes where correlated collisions are present. In terms of the dimensionless parameter $h=$ $N b_{W}^{2} \lambda_{D} \ln \left(\lambda_{D} / b_{W}\right)$, such regimes correspond to values larger than $\sim 0.1-0.2$ (see Fig. 1). In the Laplace domain, Eq. (15) involves a resonance broadening, with denominators like $s+i L_{0}+K(s)$, where the presence of $K(s)$ stems from the memory kernel. This suggests that the substitution of $Q$ by $Q_{\text {eff }}$ denotes a renormalization of the atomic energy levels induced by the correlated collisions. In practice, a calculation of the collision operator from Eq. (14) should be done by iterations. A simplification, practical for numerical applications, is provided by assuming $K(s) \simeq K\left(-i \omega_{0}\right) \equiv K_{0}$ with $\omega_{0}$ being the central frequency of the line under consideration in all denominators present in the Laplace domain. This amounts to setting $M(t) \equiv K_{0} \delta(t)$ in Eq. (15), and it leads to a simple expression for $Q_{\text {eff }}$, with a structure similar to that in the binary case Eq. (10):

$$
Q_{\text {eff }}(1, t)=e^{\left(-i L_{0}-K_{0}\right) t} \mathcal{T} \exp \left[-i \int_{0}^{t} d \tau e^{i L_{0} \tau} V\left(\mathbf{r}_{1}+\mathbf{v}_{1} \tau\right) e^{-i L_{0} \tau}\right] .
$$

\section{Application to hydrogen lines}

We have applied the collision operator formula Eq. (14) to calculations of hydrogen line shapes in ideal cases. The effective propagator has been estimated from Eq. (16). Figure 2 presents a plot of the Lyman- $\alpha$ line broadened due to ions at $N=3 \times 10^{17} \mathrm{~cm}^{-3}, T=10 \mathrm{eV}$, obtained using the unified theory (binary approximation) and compared to that obtained within the renormalized model. This case corresponds to $h=1$ and $g=0.1$. A numerical result from an ab initio simulation code [5] is also shown in the figure. The technique assumes particles moving along straight lines, in agreement with the quasi-particle model used in the collision operator model. As can be seen, the binary model overestimates the width and predicts a different shape structure, with a dip at the center. This dip is a consequence of the inadequacy of the binary model. Correlated collisions are important in the central region because they govern the average 


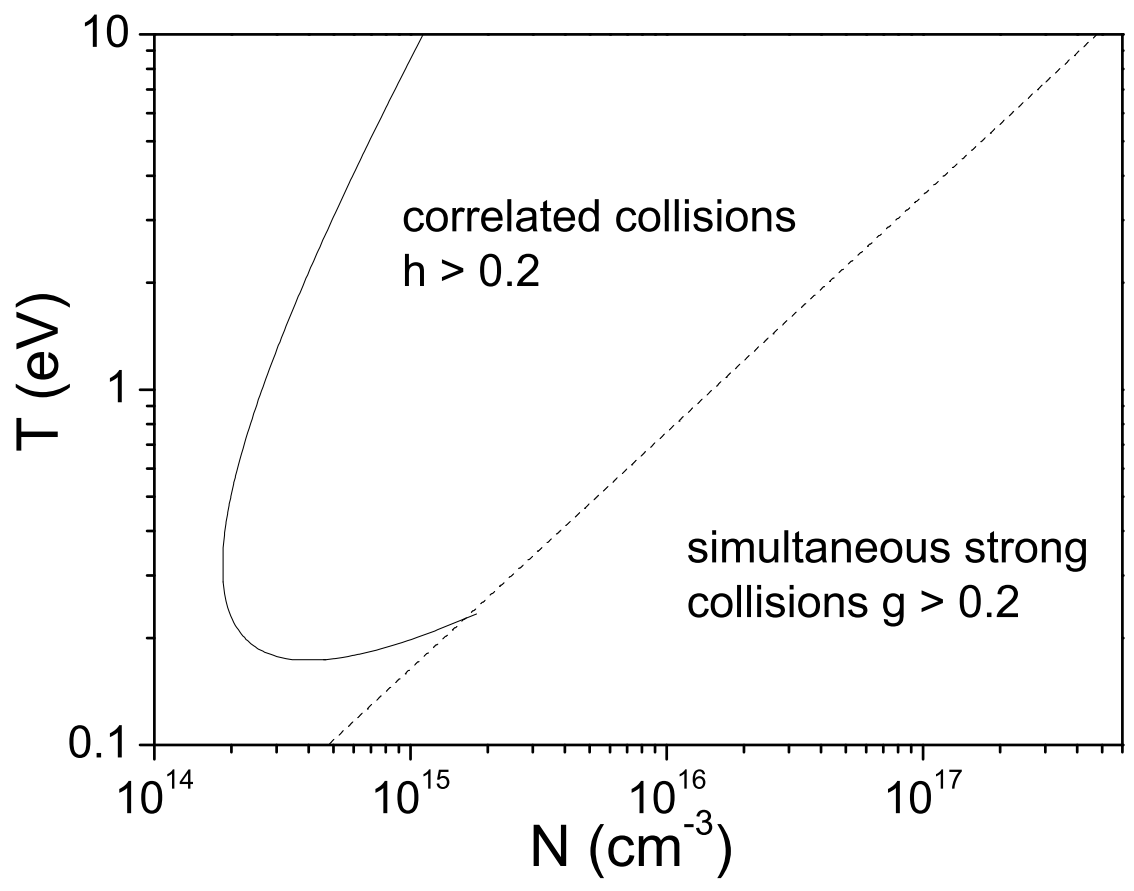

Figure 1: An illustration of the conditions where correlated collisions are present, here for hydrogen Lyman $\alpha$ in moderately dense plasmas, with ionic perturbers. The boundary is set at $h=0.2$. Also shown in the figure is the region such that simultaneous strong collisions are present, which is characterized by significant values of the $g$ parameter (see introduction). The theory is not applicable for such conditions. 
atomic evolution operator at long times, hence, by virtue of the Laplace transform, at small frequency detuning. The typical range for the dip corresponds to frequencies smaller than the matrix elements of $K$. The renormalized model gives a much better result, with no dip and with an overestimate of the width no larger than $10 \%$. The role of correlated collisions is strongly dependent on the energy level structure of the emitter. For non-degenerate energy levels (isolated lines), the Stark effect involves couplings between different energy levels, which reduces the effective range of the interaction from $\lambda_{D}$ to $v / \bar{\omega}$ (with $\hbar \bar{\omega}$ being the characteristic separation between the energy levels) [15, 16]. This reduction mitigates the effect of correlated collisions. An analysis of the role of the correlated collisions in terms of the dimensionless parameter $h$ depends on the component under consideration and is not straightforward in general. As an example we have examined the role of correlated collisions on hydrogen Lyman $\alpha$ in the presence of an external magnetic field, at $N=3 \times 10^{15} \mathrm{~cm}^{-3}, T=1$ $\mathrm{eV}$, and assuming $B=5 \mathrm{~T}$. Our calculations follow previous works performed in the framework of magnetic fusion research $[5,2,17]$. The magnetic field yields a splitting of the $2 \mathrm{p}$ level (spin is neglected), which results in a splitting of the spectral line into three components (Lorentz triplet). The broadening of the lateral components involves Stark coupling between energy levels sufficiently separated so that $v / \bar{\omega}<\lambda_{D}$ (here $\bar{\omega}=e B / 2 m_{e}$ ). On the other hand, the broadening of the central component results from the $2 \mathrm{p}_{0}-2 \mathrm{~s}$ coupling and is not affected by the degeneracy removal. Figure 3 shows the result of the calculation, for observation perpendicular to the magnetic field. The difference between the unified theory and the renormalized model on the lateral components is not as large as that on the central component.

\section{Conclusion}

We have shown that the unified theory can lead to incorrect results, with unphysical dips at the line center, if it is applied in regimes where correlated collisions are present. By applying a kinetic theory-based treatment, we have proposed an extension of the model able to account for such correlations. The resulting collision operator has a structure similar to that obtained within the unified theory. The main difference is the presence of a non-Hermitian part in the atomic Hamiltonian accounting for the correlated collisions. Applications to hydrogen lines in ideal cases, assuming neutral emitters and ion broadening, have indicated a good agreement between the model and numerical simulations. The method presented in this work provides an important correction to the usual models based on collision operators. It is applicable either to ions or to electrons. The structure of the new collision operator is convenient for physical interpretation and suitable for numerical calculations, in particular for an implementation in line shape codes that use a binary collision operator, e.g., see Refs. $[18,19,8]$. The recent Workshop on Spectral Line Shapes in Plasmas [20] provides a motivation to proceed with further investigations from the theoretical point of view, in order to create fast routines for the evaluation of spectra. Specific topics include ion dynamics, atomic structure, Zeeman effect, but also 


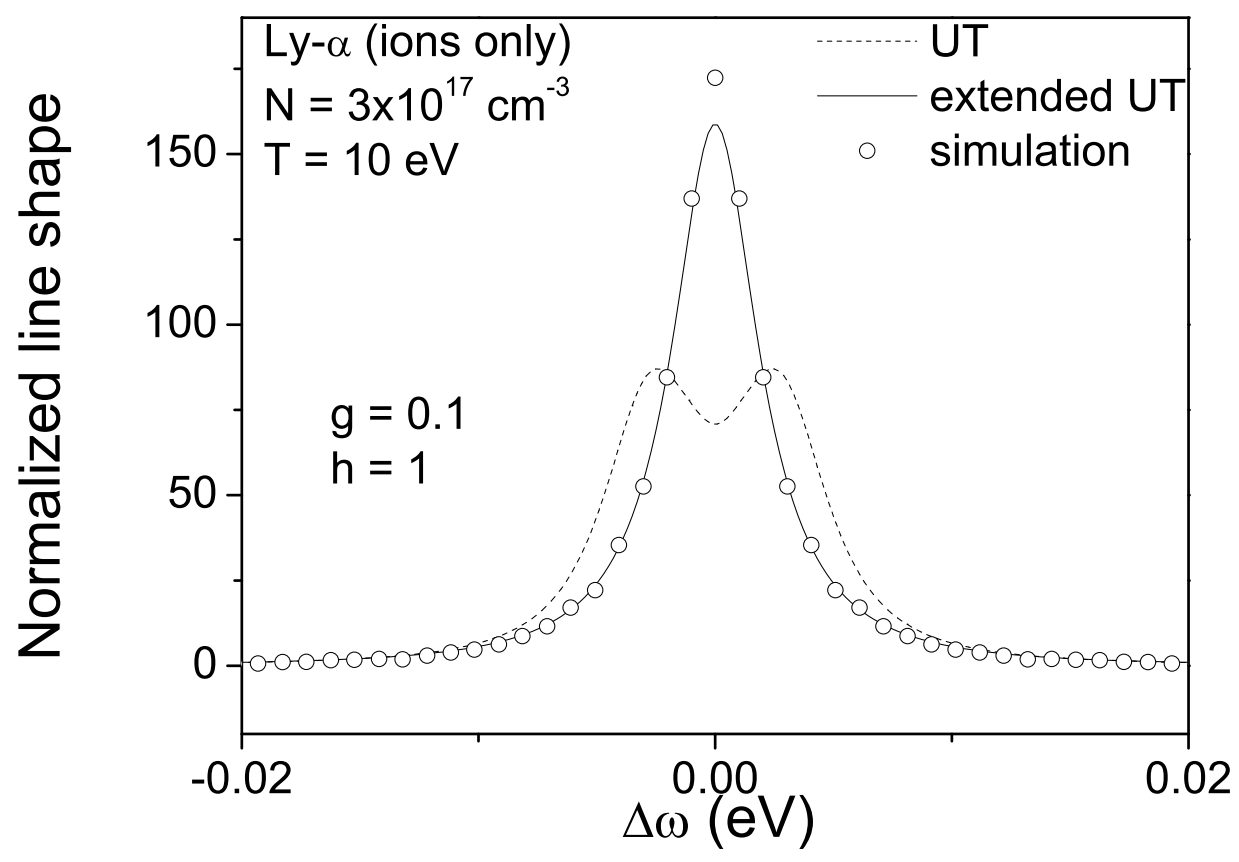

Figure 2: Profile of Ly- $\alpha$ at $N=3 \times 10^{15} \mathrm{~cm}^{-3}, T=1 \mathrm{eV}$. This case corresponds to a regime where correlated collisions are present. The binary model overestimates the width and predicts a different shape, with a dip at the center. The renormalized model gives a much better result, with no dip and with an overestimate no larger than $10 \%$. 


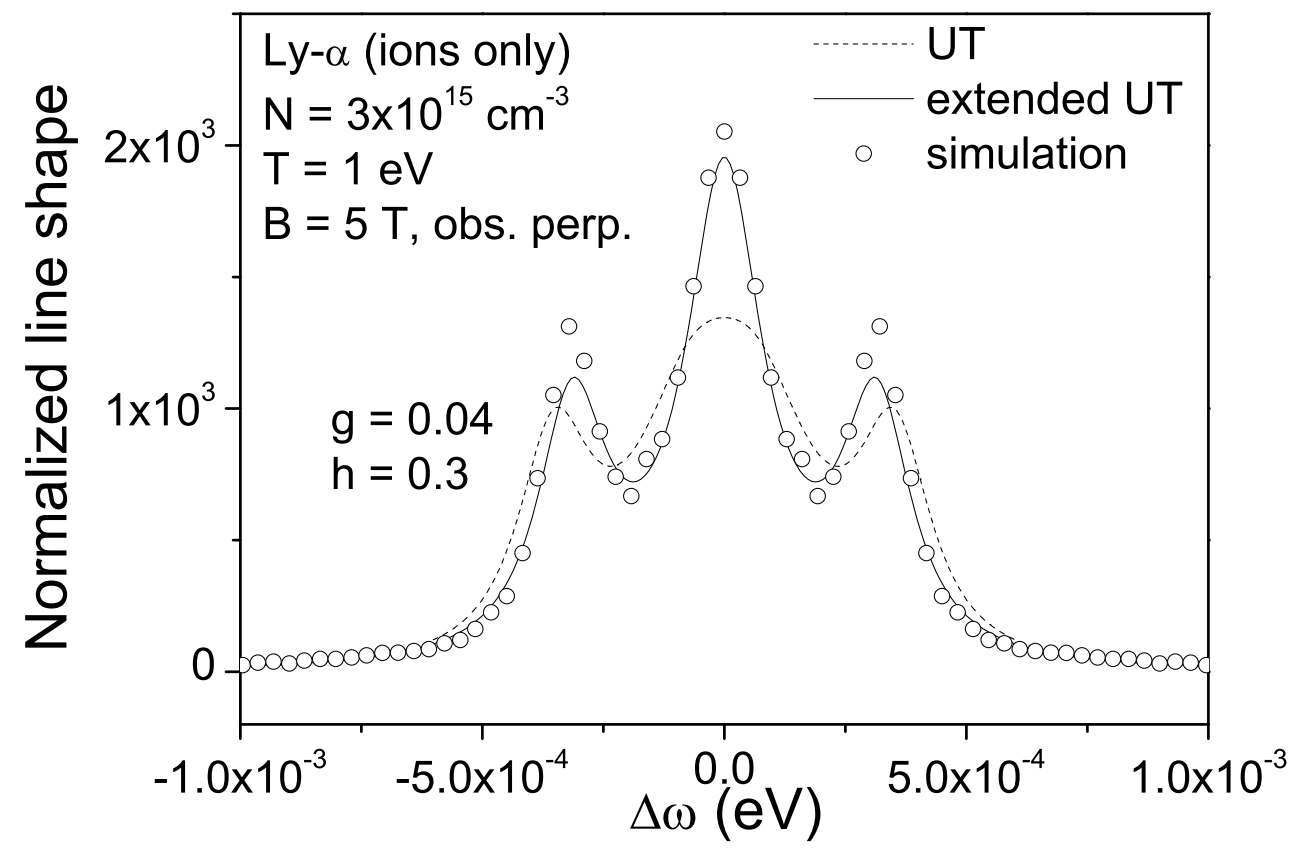

Figure 3: Profile of Ly- $\alpha$ in the presence of an external magnetic field. The spectral line is split due to the Zeeman effect. The lateral components are not much affected by correlated collisions because the broadening involves Stark coupling between non-degenerate levels. Here, the result $h=0.3$ concerns the central component. 
more advanced concepts such as penetrating collisions or perturber-perturber correlation effects. Moreover, further work could address the description of two-photon processes and the corresponding redistribution functions [21].

\section{Acknowledgements}

This work was carried out within the framework of the European Fusion Development Agreement and the French Research Federation for Fusion Studies. It is supported by the European Communities under the contract of Association between Euratom and CEA. The views and opinions expressed herein do not necessarily reflect those of the European Commission.

\section{References}

[1] C. A. Iglesias et al., High Energy Density Phys. 5, 97 (2009)

[2] J. Rosato, V. Kotov, and D. Reiter, J. Phys. B: At. Mol. Opt. Phys. 43, $144024(2010)$

[3] R. W. Lee, J. Phys. B: Atom. Molec. Phys. 12, 1145 (1979)

[4] R. Stamm and D. Voslamber, J. Quant. Spectrosc. Radiat. Transfer 22, 599 (1979)

[5] J. Rosato et al., Phys. Rev. E 79, 046408 (2009)

[6] S. Alexiou, High Energy Density Phys. 5, 225 (2009)

[7] E. Stambulchik and Y. Maron, High Energy Density Phys. 6, 9 (2010)

[8] S. Ferri et al., Phys. Rev. E 84, 026407 (2011)

[9] D. Voslamber, Z. Naturforsch. 24a, 1458 (1969)

[10] E. W. Smith, J. Cooper, and C. R. Vidal, Phys. Rev. 185, 140 (1969)

[11] J. Rosato, H. Capes, and R. Stamm, Phys. Rev. E 86, 046407 (2012)

[12] H. Capes and D. Voslamber, Phys. Rev. A 5, 2528 (1972)

[13] H. Capes and D. Voslamber, Phys. Rev. A 15, 1751 (1977)

[14] R. Balescu, Equilibrium and Nonequilibrium Statistical Mechanics (WileyInterscience, New York, 1975)

[15] H. R. Griem et al., Phys. Rev. 125, 177 (1962)

[16] H. R. Griem, M. Blaha, and P. C. Kepple, Phys. Rev. A 19, 2421 (1979)

[17] J. Rosato et al., High Energy Density Phys. 5, 93 (2009) 
[18] R. Mancini et al., Computer Physics Comm. 63, 314 (1991)

[19] M. L. Adams et al., Phys. Rev. E 66, 066413 (2002)

[20] E. Stambulchik, these proceedings

[21] D. Voslamber and J.-B. Yelnik, Phys. Rev. Lett. 41, 1233 (1978) 